\title{
Unrelated donor umbilical cord blood transplantation for the treatment of hematologic malignancies
}

\author{
Craig Sauter and Juliet N. Barker \\ Adult Allogeneic Bone Marrow Transplantation Service, Memorial Sloan-Kettering Cancer Center, \\ New York, New York, USA
}

\begin{abstract}
Purpose of review-This review summarizes the state of the art of unrelated donor (URD) umbilical cord blood transplantation (UCBT) for the treatment of hematologic malignancies and discusses the current issues associated with the use of this hematopoietic stem cell (HSC) source.

Recent findings - In contrast to the very high transplant-related mortality associated with the early experience of UCBT, recent series have been associated with comparable survival to that of human leucocyte antigen-matched URD transplantation in children with similarly promising results in adults with the use of double-unit grafts. In addition, utilization of reduced-intensity conditioning regimens has been successful extending access to patients unsuitable for myeloablation. Consequently, the use of umbilical cord blood as a HSC source and the global inventory of units in public banks is rapidly increasing although challenges associated with engraftment, unit quality, and infectious complications remain and will be discussed in this review.

Summary-URD umbilical cord blood is an alternative HSC source offering a unique set of advantages and disadvantages as compared with the transplantation of HSC from unrelated volunteers. Improved transplant outcomes are now making UCBT a rival to URD transplantation for the treatment of hematologic malignancies.
\end{abstract}

\section{Keywords}

allogeneic transplantation; cord blood; hematologic malignancy

\section{Introduction}

\begin{abstract}
Allogeneic hematopoietic stem cell transplantation (HSCT) is indicated for the treatment of many high-risk hematologic malignancies. Unfortunately, application of this treatment is limited by a lack of suitable donors. Only $25 \%$ of patients have a HLA-matched sibling donor suitable for HSC donation and despite the many millions of volunteer donors registered in the unrelated donor (URD) pool, many patients do not have an adequately human leucocyte antigen (HLA)-matched URD using high-resolution donor-recipient HLA matching especially patients from racial and ethnic minorities [1]. Additionally, the procurement of an URD graft can often take months, whereas patients with hematologic malignancies frequently require urgent transplantation.
\end{abstract}

(C) 2008 Wolters Kluwer Health | Lippincott Williams \& Wilkins

Correspondence to Juliet N. Barker, MBBS (Hons), FRACP, Attending Physician, Adult Allogeneic Bone Marrow Transplantation Service, Memorial Sloan-Kettering Cancer Center, 1275 York Avenue, New York, NY 10065, USA Tel: +1 212 639 3468; fax: +1 212717 3500; barkerj@mskcc.org. 
Umbilical cord blood (UCB) can reconstitute hematopoiesis in adults following both myeloablative $[2-8]$ and reduced-intensity/nonmyeloablative (NMA) $\left[9,10^{\circ}, 11\right]$ conditioning and has the advantage of ready availability. Importantly, in marked contrast to the transplantation of URD HSC [12], the reduced stringency of the required HLA match with UCBT translates to the potential to extend allogeneic HSCT access to patients without other suitable donors (Table 1). This factor alone, along with multiple other attributes, frequently outweighs the disadvantages of UCBT as compared with URD HSCT (summarized in Table 2), thus accounting for the rapid expansion of use of this relatively new HSC source (Fig. 1). This review will outline the current status of UCBT as compared with URD HSC transplantation as well as discussing current issues associated with the transplantation of this HSC source.

\section{Outcome of umbilical cord blood transplantation compared with unrelated donor bone marrow transplantation}

Although no randomized controlled trials have compared UCBT and URD transplantation, retrospective studies have compared single-unit UCBT with URD bone marrow transplantation (BMT) using myeloablative conditioning in adults and children. In 2004, Laughlin et al. [5] and Rocha et al. [6] reported the first comparisons between UCBT and URD transplantation in adults. The American series found comparable survival after UCBT $(n=150)$ and one antigen-mismatched BMT $(n=83)$ [5], whereas the Europeans reported that HLA-mismatched adult UCBT $(n=98)$ was associated with comparable survival to $6 / 6$ HLA antigen-matched BMT $(n=584)$ [6]. In contrast, a Japanese series reported by Takahashi et al. [7] demonstrated superior transplant-related mortality (TRM) and diseasefree survival (DFS) in 68 adult UCBT as compared with 45 URD BMT recipients.

More recently, Eapen et al. [13*0] have analyzed the outcomes of 503 UCBT recipients of 4 6/6 HLA-A, B antigen and DRB1 allele-matched single-unit UCBT as compared with those of URD BMT in children below 16 years of age with leukemia. Most notably, in a subset analysis comparing UCBT outcomes with the 116 recipients of the 'gold standard' of $8 / 8$ HLA allele-matched bone marrow, the 35 6/6 HLA-matched UCBT recipients had significantly higher 5-year DFS, with $2015 / 6$ and 267 4/6 UCBT having comparable DFS with that of $8 / 8$ allele-matched BMT recipients and demonstrated a robust protection against relapse (Table 3).

These findings support UCBT as an alternative to URD BMT in children. Further, if engraftment after UCBT is improved, it suggests that pediatric UCBT may be a superior HSC for the treatment of leukemia. In adults, the American and European comparisons, although establishing UCBT as a potential alternative to URD BMT, have highlighted that the poor engraftment and high TRM must be addressed for this HSC to be widely adopted. At the current time, whether UCBT will be offered to a patient will be frequently determined by the relative availability of a closely HLA-matched (7-8/8 alleles) URD versus an UCB graft of at least 4/6 HLA-A, B antigen and DRB 1 allele match and adequate dose; and the experience and research bias of the transplant center.

\section{Strategies to optimize engraftment and reduce transplant-related mortality}

Graft failure is a major risk associated with UCBT and from early in the practice of UCBT it was recognized that the total nucleated cell (TNC) dose $[2,3]$ and the infused CD34+ dose [4] per kilogram recipient body weight were significant determinants of sustained donor engraftment. Investigation of ex-vivo expansion [14], coinfusion of T-cell depleted haploidentical cells (to bridge the neutropenic period until the engraftment of a T-replete UCB unit) [15], infusion of mesenchymal stem cells [16], intra-bone marrow injection [17], 
and agents to augment UCB homing [18] to improve both overall UCB engraftment and the speed of neutrophil recovery is ongoing. However, perhaps the simplest strategy to augment engraftment pioneered by the University of Minnesota is the infusion of a double-unit graft. Although traditionally thought of as being only needed for adult UCBT recipients this approach is equally as relevant to many children given graft failure is still a devastating feature of many pediatric UCBT series and many larger children will only have access to units of relatively low cell doses that similarly challenge adult UCBT recipients.

Initial investigation with double-unit UCBT following a total-body irradiation (TBI)-based myeloablative conditioning regimen yielded a DFS of 57\% [95\% confidence interval (CI) $35-79$ ) in 23 leukemia patients (median age 24 years), with a DFS of $72 \%$ if transplanted in remission [19]. Updated survival data after myeloablative double-unit UCBT in high-risk hematologic malignancies is shown in Fig. 2. Interestingly, both engraftment and survival was improved after double-unit UCBT as compared with historical single-unit controls [4] despite only one of two relatively low cell dose units being responsible for sustained donor engraftment in the vast majority of patients. This raises the possibility that the 'losing' unit is somehow facilitating the engraftment of the engrafting or 'winning' unit. However, it is important to note that the single-unit historical controls were transplanted using cyclophosphamide and TBI with antithymocyte globulin (ATG) and cyclosporine-A (CSA)/ methylprednisolone as immunosuppression. In contrast, though the double-unit transplants were also performed with cyclophosphamide/TBI and CSA, the ATG and MP were substituted with low-dose fludarabine (Flu) and mycophenolate mofetil (MMF). This raises the possibility that some of the advantage of double-unit UCBT was due to changes in the preparative regimen and immune suppression independent of the graft. This question is therefore being investigated in the Bone Marrow Transplant Clinical Trials Network (BMT CTN) single versus double-unit randomized trial in children in the United States utilizing the cyclophosphamide/Flu/TBI and CSA/MMF regimen. Importantly, however, this study may not fully answer the question of the utility of double-unit UCBT in adults. A major question for the field currently is how double-unit UCBT compares with URD peripheral blood stem cell (PBSC) transplantation in adult patients and should be studied in the near future.

The double-unit UCBT experience has raised unique questions about transplant biology especially given that preliminary University of Minnesota data have suggested that this strategy has also been associated with a reduced risk of relapse as compared with single-unit UCBT [20]. To date, no reliable factor has been able to predict which unit will predominate in engraftment after double-unit transplantation. Interestingly, Scaradavou et al. [21] have recently demonstrated an association between UCB unit CD34+ cell viability after thaw [as measured by flow cytometric 7-amino-actinomycin D (7-AAD) staining] and unit predominance. In this analysis of 26 double-unit UCBTs, although the factor determining unit predominance when both units of a double-unit graft have high viability was unclear, units with low viability did not engraft $(P=0.007)$. Such data suggest that the reason that double-unit UCBT is efficacious may simply be because it increases the chance that the patient will receive at least one unit of high viability and thus with engraftment potential. This introduces the concept that postthaw CD34+ cell viability could be an effective measure of unit quality and has the advantage that, unlike colony-forming assays, is available on the day of transplant. Postthaw unit quality is a relatively new variable to be considered in the field of UCBT and will be a critical area of investigation for the future. If these findings are confirmed it would suggest that double-unit UCBT may be indicated even in children given the viability of a unit that appears satisfactory from the standpoint of HLAmatched and TNC dose cannot be predicted prior to thaw.

Although the poor engraftment and high TRM associated with low-infused TNC dose in single-unit UCBT has understandably led to a focus on strategies to augment graft cell dose, 
unit selection is complicated by the fact that both engraftment and TRM are also influenced by HLA match. For example, in an analysis of 989 single-unit myeloablative UCBT recipients facilitated by the National Cord Blood Program of the New York Blood Center (NYBC), HLA-A, B antigen and DRB1 allele match was associated with significantly improved engraftment, a lower incidence of severe acute graft-versus-host disease (GVHD), lower TRM, and improved DFS [22]. Eurocord analyses have also found that HLA match is associated with significantly improved engraftment and lower TRM [8].

These findings lead to the question of how to 'trade-off' HLA match with TNC dose when selecting UCB units for transplantation. Although this issue is yet to be fully resolved it is intriguing that in the NYBC analysis referenced above [22] 6/6 HLA-matched UCBT recipients (any dose) had superior DFS to recipients of either $5 / 6$ units at least $2.5 \times 10^{7}$ $\mathrm{TNC} / \mathrm{kg}$ or $4 / 6$ units at least $5 \times 10^{7} \mathrm{TNC} / \mathrm{kg}$. Further, recipients of $5 / 6$ at least $2.5 \times 10^{7}$ $\mathrm{TNC} / \mathrm{kg}$ units had a comparable DFS to those of $4 / 6$ HLA-matched units with a TNC at least $5.0 \times 10^{7} / \mathrm{kg}$ [although with less severe acute GVHD (aGVHD)]. This raises the concept of a 'sliding scale' in unit selection with HLA-matched compensating for lesser cell dose (or conversely that the greater the HLA mismatch the greater the cell dose required), and prompts a unit selection algorithm of $6 / 6$ units followed by $5 / 6$ units above $2.5 \times 10^{7} / \mathrm{kg}$, and $4 / 6$ units above $5.0 \times 10^{7} / \mathrm{kg}$. However, many patients will not have access to such units, and some patients with such optimal units will still not engraft. One strategy to address this limitation that is being investigated in a Center for Bone Marrow Transplant Research (CIBMTR) sponsored study is to prioritize HLA match above a cell dose threshold of $1.5 \times$ $10^{7} / \mathrm{kg}$ but to augment engraftment by the infusion of two units.

\section{Graft-versus-host disease after umbilical cord blood transplantation}

Although GVHD remains one of the leading causes of TRM in allogeneic HSCT, UCBT has consistently demonstrated a lower than expected incidence of acute and chronic GVHD (cGVHD) [2-8] especially given the considerable degree of HLA mismatch if highresolution typing is considered [23]. In comparison with URD BMT Eapen et al. [13**] reported a similar incidence of grade $2-4$ acute and chronic GVHD in pediatric 8/8 allelematched BMT and 4-6/6 A, B antigen, DRB1-matched UCBT. In adult recipients, Laughlin et al. [5] found a similar incidence of grade 2-4 aGVHD and a lesser incidence of extensive cGVHD as compared with HLA-matched BMT recipients. In contrast, Rocha et al. [6] reported a lower risk of grade $2-4$ aGVHD in adult UCBT as compared with HLA-matched BMT recipients with a relative risk of cGVHD of 0.64 after UCBT although this did not reach significance $(P=0.11)$. Takahashi et al. [7] have reported similar findings to the Rocha et al. study [6], and more recently these investigators have even reported a significantly lower incidence of grade 3-4 aGVHD and extensive cGVHD after predominantly HLA-matched-related donor HSC transplantation and mismatched URD donor UCBT in adults [24"].

Of further interest beyond the incidence of GVHD is the nature of this disease after UCBT and its response to therapy. Although this has not yet been examined for aGVHD, Arora et al. $\left[25^{\circ}\right]$ found more frequent responses of cGVHD to therapy in $47 \mathrm{UCBT}$ as compared with predominantly HLA-matched URD BMT recipients at 2 months (74 versus $48 \%, P=$ 0.005 ), 6 months ( 78 versus $49 \%, P=0.001$ ) and 1 year ( 72 versus $51 \%, P=0.03$ ) following cGVHD diagnosis. UCBT cGVHD was also associated with a lower TRM (11 versus $27 \%$ with URD BMT). It is likely that the findings in this study in favor of UCBT may have even been more pronounced if the URD transplant recipients had received PBSC as the HSC source rather than bone marrow, and given the wide adoption of PBSC (Fig. 1) comparisons of both aGVHD and cGVHD after UCBT to recipients of URD PBSC should be a priority for the future. 
The exact reasons for the relatively low incidence of GVHD after UCBT are unknown but likely result from the functional immaturity of the infused lymphocytes including decreased cytotoxicity, an altered cytokine profile, decreased HLA expression and increased regulatory $\mathrm{T}$ cells. Of even more interest is to understand the biology of why UCBT is associated with a retained graft-versus-leukemia effect despite the GVHD reduction.

\section{Infectious complications after umbilical cord blood transplantation and immune recovery}

Opportunistic infections are a significant cause of TRM in HSCT regardless of graft source. However, studies have revealed varying results in the assessment of infection risk after UCBT as compared with other HSC sources. A University of Minnesota analysis revealed equal incidences of one or more serious infections in unmodified bone marrow [81\% (95\% CI 65-97)], T-cell depleted [83\% (95\% CI 60-100)] and UCB [90\% (95\% CI 74-100)] pediatric transplant recipients $(P=0.48)$ in the first 2 years after transplant, with no significant differences overall when taking all serious infections into account [26]. Further, more recently this group has reported a similar risk of cytomegalovirus (CMV) infection in recipients of UCB, bone marrow and PBSC grafts [27]. Another study [28] has reported increased incidences of severe infection in 48 adult UCBT ( $85 \%$ risk) as compared with 144 adults URD HSC transplant (69\% risk) recipients, although day 100 and 3-year infectionrelated mortality did not differ between HSC sources.

Regardless of the specifics of such comparisons infection is a major challenge in UCBT and at many centers infection-related mortality is now the most frequent cause of death in UCBT with the majority of deaths occurring within the first 3-4 months $\left[2^{\circ}\right]$. Although improved engraftment with new preparative regimens and double-unit grafts, and aggressive supportive care to abrogate neutropenic sepsis and prevent fungal infections by the use of extended spectrum azoles have led to decreased infection-related TRM, viral infections remain a critical challenge in the early postengraftment period. For example, Duke University analyzed 330 pediatric patients undergoing UCBT and reported most deaths within the first 6 months after transplant being attributable to opportunistic infection, of which more than half were secondary to CMV or adenovirus [29 $\left.{ }^{\circ}\right]$.

Important in interpreting the infectious complications and immune recovery seen after UCBT is not only considering patient and unit characteristics but also what preparative regimen and immune suppression was used. The use of ATG [30,31], corticosteroids, or both for GVHD prophylaxis, for example, appears to be associated with impaired immune recovery and increased risk of severe infection. How to augment immune reconstitution is a major question in the field of UCBT today and assume even greater importance with the recognition that improved immune recovery has also been associated with protection against leukemic relapse [32]. Cellular therapy approaches, although clearly challenging given the naïve neonatal immune system, may yet show promise in the future. In the interim, improved preparative regimens/immune suppression and aggressive supportive care including surveillance for viral reactivation is mandatory in the care of UCBT patients in the early posttransplant period.

\section{Reduced-intensity or nonmyeloablative conditioning}

Reduced-intensity conditioning (RIC) or NMA HSCT has been investigated as a method to offer the potential benefit of a graft-versus-malignancy effect to older, more heavily pretreated, more infirm patients, or all with less toxicity. Early series from the University of Minnesota demonstrated that UCBT after NMA conditioning was feasible [9]. However, it was observed that there was a strong association between recent exposure to combination 
chemotherapy or a prior autologous transplant and the likelihood of sustained donor engraftment (Fig. 3) [33]. This group has recently updated their NMA UCBT experience $\left[10^{\circ}\right]$. One-hundred and ten patients (median age 51 years) with high-risk or advanced leukemias, myelodysplasia and Hodgkin's or non-Hodgkin's lymphoma unsuitable for myeloablative conditioning received cyclophosphamide $50 \mathrm{mg} / \mathrm{kg}$, Flu $200 \mathrm{mg} / \mathrm{m}^{2}$, and TBI $200 \mathrm{cGy}$ with immune suppression of CSA/MMF. Eighty-five percentage of patients received double-unit grafts to attain a target TNC dose of at least $3.0 \times 10^{7} / \mathrm{kg}$. In this highrisk patient group, TRM was $26 \%(95 \% \mathrm{CI} 18-34)$ at 3 years with an overall survival of $45 \%$ (95\% CI 34-56) and progression-free survival (PFS) of 38\% (95\% CI 28-48) at 3 years. Interestingly, the PFS was significantly higher in recipients of double-unit [39\% (95\% CI 27-51)] as compared with single-unit [24\% (95\% CI 4-44)] grafts. Further to these findings, the Minnesota group has also reported comparable PFS after RIC allograft in recipients older than 55 years of matched-related donor $(n=47)$ or UCB $(n=43)$ grafts with 3 -year PFS of 30\% (95\% CI 16-44) and 34\% (95\% CI 19-48), respectively [34].

Ballen et al. [11] have also investigated double-unit UCBT utilizing a RIC regimen of Flu $180 \mathrm{mg} / \mathrm{m}^{2}$ with melphalan $100 \mathrm{mg} / \mathrm{m}^{2}$, rabbit ATG, and CSA/MMF in advanced hematologic malignancies or severe aplastic anemia reporting a 100-day TRM of $14 \%$ and a promising 1-year DFS of $67 \%$ in 21 patients. The outcomes from these series appear comparable to previously published series of RIC/NMA transplantation using volunteer donors but this will need to be studied formerly in randomized studies in the future. For the meantime, major questions in the field of RIC/NMA UCBT are: how to ensure engraftment in patients (such as those with myelodysplasia, myelofibrosis and acute myelogenous leukemia who have only received a single induction) without intensive prior chemotherapy (especially given the addition of ATG to the NMA preparative regimen as a strategy to augment engraftment is associated with a high incidence of Epstein-Barr virus posttransplant lymphoproliferative disease [30]); and what is the efficacy of RIC/NMA UCBT in specific disease entities.

\section{Umbilical cord blood banking}

As a counterpart to the increased adoption of UCB as an alternative HSC source, the number of units banked worldwide continues to increase with at least 250000 units for unrelated recipient use banked to date. However, the UCB search continues to be a challenge with no centralized search mechanism to access all units in the global inventory (Fig. 4) and no international regulation to ensure uniform standards from bank to bank. Notably, it is not known how many units would be needed to ensure, for example, a 5/6 HLA-A, B antigen and DRB1 allele-matched unit for the majority of patients of any race or ethnicity. Such projections, although complicated, are important in the consideration of the future funding needed for public UCB banks. A further issue is that of unit quality including: what are the critical determinants of a quality product; and how this should be regulated. McCullough et al. [35] investigated the quality of 268 units from banks in the United States and Europe and discovered that quality issues existed in $56 \%$ of units, with $10 \%$ likely and $35 \%$ potentially associated with patient risk. The major issues associated with UCB banking are discussed by Atlas [36] and Rubinstein [37].

\section{Conclusion}

UCB is a promising alternative HSC source although reaching its full potential will likely require a significant increase in the size of the global UCB inventory. If that can be achieved, this combined with measures such as improved preparative regimens, double-unit grafts, improved supportive care, measures to augment immune recovery will likely improve 
TRM and thus extend the adoption of UCBT to treat patients with high-risk hematologic malignancies.

\section{References and recommended reading}

Papers of particular interest, published within the annual period of review, have been highlighted as:

- of special interest

•• of outstanding interest

Additional references related to this topic can also be found in the Current World Literature section in this issue (p. 640).

1. Kollman C, Abella E, Baitty R. Assessment of optimal size and composition of the US National Registry of hematopoietic stem cell donors. Transplantation. 2004; 78:89-95. [PubMed: 15257044]

2. Rubinstein P, Carrier C, Scaradavou A, et al. Outcomes among 562 recipients of placental-blood transplants from unrelated donors. N Engl J Med. 1998; 339:1565-1577. [PubMed: 9828244]

3. Gluckman E, Rocha V, Boyer-Chammard A, et al. Outcome of cord-blood transplantation from related and unrelated donors. Eurocord Transplant Group and the European Blood and Marrow Transplantation Group. N Engl J Med. 1997; 337:373-381. [PubMed: 9241126]

4. Wagner JE, Barker JN, DeFor TE, et al. Transplantation of unrelated donor umbilical cord blood in 102 patients with malignant and nonmalignant diseases: influence of CD34 cell dose and HLA disparity on treatment-related mortality and survival. Blood. 2002; 100:1611-1618. [PubMed: 12176879]

5. Laughlin MJ, Eapen M, Rubinstein P, et al. Outcomes after transplantation of cord blood or bone marrow from unrelated donors in adults with leukemia. N Engl J Med. 2004; 351:2265-2275. [PubMed: 15564543]

6. Rocha V, Labopin M, Sanz G, et al. Transplants of umbilical-cord blood or bone marrow from unrelated donors in adults with acute leukemia. N Engl J Med. 2004; 351:2276-2285. [PubMed: 15564544]

7. Takahashi S, Iseki T, Ooi J, et al. Single-institute comparative analysis of unrelated bone marrow transplantation and cord blood transplantation for adult patients with hematologic malignancies. Blood. 2004; 104:3813-3820. [PubMed: 15280199]

8. Gluckman E, Rocha V. Donor selection for unrelated cord blood transplants. Curr Opin Immunol. 2006; 18:565-570. [PubMed: 16893632]

9. Barker JN, Weisdorf DJ, DeFor TE, et al. Rapid and complete donor chimerism in adult recipients of unrelated donor umbilical cord blood transplantation after reduced-intensity conditioning. Blood. 2003; 102:1915-1919. [PubMed: 12738676]

10 • Brunstein C, Barker JN, Weisdorf DJ, et al. Umbilical cord blood transplantation after nonmyeloablative conditioning: impact on transplant outcomes in 110 adults with hematological disease. Blood. 2007; 110:3064-3070. [PubMed: 17569820] This study represents the largest series of UCBT using NMA conditioning.

11. Ballen KK, Spitzer TR, Yeap BY, et al. Double unrelated reduced-intensity umbilical cord blood transplantation in adults. Biol Blood Marrow Transplant. 2007; 13:82-89. [PubMed: 17222756]

12. Lee SJ, Klein J, Haagenson M, et al. High-resolution donor-recipient HLA matching contributes to the success of unrelated donor marrow transplantation. Blood. 2007; 110:4576-4583. [PubMed: 17785583]

13 ••. Eapen M, Rubinstein P, Zhang M. Outcomes of transplantation of unrelated donor umbilical cord blood and bone marrow in children with acute leukaemia: a comparison study. Lancet. 2007; 369:1947-1954. [PubMed: 17560447] This is a landmark study demonstrating that UCB is an acceptable alternative HSC source for the transplantation of children with leukemia with comparable survival after 4-5/6 HLA-matched UCBT as compared with the gold standard of 8/8 allele-matched BMT and superior survival after 6/6-matched UCBT. 
14. de Lima M, McMannis J, Gee A, et al. Transplantation of ex vivo expanded cord blood cells using the copper chelator tetraethylenepentamine: a phase I/II clinical trial. Bone Marrow Transplant. 2008; 41:771-778. [PubMed: 18209724]

15. Magro E, Regidor C, Cabrera R, et al. Early hematopoietic recovery after single unit unrelated cord blood transplantation in adults supported by coinfusion of mobilized stem cells from a third party donor. Haematologica. 2006; 91:640-648. [PubMed: 16670070]

16. MacMillan M, Ramsey N, Atkinson K, Wagner J. Ex-vivo culture expanded parental haploidentical mesenchymal stem cells (MSC) to promote engraftment in recipients of unrelated donor umbilical cord blood (UCB): results of a phase I-II clinical trial. Blood. 2002; 100:836a.

17. Ibatici A, Raiola A, Podesta M. Direct intra-bone injection of unrelated cord blood cells overcomes the problem of delayed engraftment and improves the feasibility of hematopoietic transplant in adult patients. Blood. 2007; 110:105a.

18. Christopherson KW 2nd, Paganessi LA, Napier S, et al. CD26 inhibition on CD34+ or lineagehuman umbilical cord blood donor hematopoietic stem cells/hematopoietic progenitor cells improves long-term engraftment into NOD/SCID/Beta2null immunodeficient mice. Stem Cells Dev. 2007; 16:355-360. [PubMed: 17610365]

19. Barker JN, Weisdorf DJ, DeFor TE, et al. Transplantation of two partially HLA-matched umbilical cord blood units to enhance engraftment in adults with hematologic malignancy. Blood. 2005; 105:1343-1347. [PubMed: 15466923]

20. Verneris MR, Brunstein C, DeFor TE, et al. Risk of relapse after umbilical cord blood transplantation (UCBT) in patients with acute leukemia: marker reduction in recipients of two units. Blood. 2005; 106:93a.

21. Scaradavou A, Smith K, Hawke R, et al. CD34+ viability is a critical determinant of the engraftment potential of umbilical cord blood (UCB) in double unit transplantation. Blood. 2007; 110:600a.

22. Barker JN, Scaradavou A, Stevens C, Rubinstein P. The dose-match interaction in umbilical cord blood (UCB) transplantation: an analysis of the impact of cell dose and HLA-match on the disease-free survival (DFS) of 989 patients transplanted with single units for hematologic malignancy. Blood. 2007; 110:105a.

23. Kogler G, Enczmann J, Rocha V, et al. High-resolution HLA typing by sequencing for HLA-A, -B, -C, -DR, -DQ in 122 unrelated cord blood/patient pair transplants hardly improves long-term clinical outcome. Bone Marrow Transplant. 2005; 36:1033-1041. [PubMed: 16247425]

$24 \cdot$ Takahashi S, Ooi J, Tomonari A, et al. Comparative single-institute analysis of cord blood transplantation from unrelated donors with bone marrow or peripheral blood stem-cell transplants from related donors in adult patients with hematologic malignancies after myeloablative conditioning regimen. Blood. 2007; 109:1322-1330. [PubMed: 17038536] This is an interesting analysis comparing URD UCBT with related donor transplantation possibly representing a paradigm shift in the field.

25 • Arora M, Nagaraj S, Wagner JE, et al. Chronic graft versus host disease following unrelated donor hematopoietic stem cell transplantation: higher response rate in recipients of unrelated donor umbilical cord blood. Biol Blood Marrow Transplant. 2007; 13:1145-1152. [PubMed: 17889350] This is the first report introducing the concept of differing response to treatment of cGVHD demonstrating an advantage with UCBT as compared with URD BMT.

26. Barker JN, Hough RE, van Burik JA, et al. Serious infections after unrelated donor transplantation in 136 children: impact of stem cell source. Biol Blood Marrow Transplant. 2005; 11:362-370. [PubMed: 15846290]

27. Walker CM, van Burik JA, De For TE, et al. Cytomegalovirus infection after allogeneic transplantation: comparison of cord blood with peripheral blood and marrow graft sources. Biol Blood Marrow Transplant. 2007; 13:1106-1115. [PubMed: 17697973]

28. Parody R, Martino R, Rovira M, et al. Severe infections after unrelated donor allogeneic hematopoietic stem cell transplantation in adults: comparison of cord blood transplantation with peripheral blood and bone marrow transplantation. Biol Blood Marrow Transplant. 2006; 12:734748. [PubMed: 16785063]

29 •. Szabolcs P, Niedzwiecki D. Immune reconstitution after unrelated cord blood transplantation. Cytotherapy. 2007; 9:111-122. [PubMed: 17453963] This study is an excellent comprehensive 
review of the unique characteristics of immune reconstitution in UCBT and highlights the challenge of infectious complications in UCBT especially in the postengraftment period.

30. Brunstein CG, Weisdorf DJ, Defor T, et al. Marked increased risk of Epstein-Barr virus-related complications with the addition of antithymocyte globulin to a nonmyeloablative conditioning prior to unrelated umbilical cord blood transplantation. Blood. 2006; 108:2874-2880. [PubMed: 16804113]

31. Robin M, Marque-Juillet S, Scieux C, et al. Disseminated adenovirus infections after allogeneic hematopoietic stem cell transplantation: incidence, risk factors and outcome. Haematologica. 2007; 92:1254-1257. [PubMed: 17666361]

32. Parkman R, Cohen G, Carter SL, et al. Successful immune reconstitution decreases leukemic relapse and improves survival in recipients of unrelated cord blood transplantation. Biol Blood Marrow Transplant. 2006; 12:919-927. [PubMed: 16920557]

33. Barker JN, Weisdorf DJ, Defor TE, et al. Non-myeloablative umbilical cord blood transplantation (UCBT): low transplant-related mortality in 59 high-risk adults. Blood. 2004; 104:235a.

34. Majhail NS, Brunstein CG, Tomblyn M, et al. Reduced-intensity allogeneic transplant in patients older than 55 years: unrelated umbilical cord blood is safe and effective for patients without a matched related donor. Biol Blood Marrow Transplant. 2008; 14:282-289. [PubMed: 18275894]

35. McCullough J, McKenna D, Kadidlo D, et al. Issues in the quality of umbilical cord blood stem cells for transplantation. Transfusion. 2005; 45:832-841. [PubMed: 15934980]

36. Atlas LD. The National Marrow Donor Program in 2006: constants and challenges. Transfusion. 2006; 46:1080-1084. [PubMed: 16836552]

37. Rubinstein P. Why cord blood? Hum Immunol. 2006; 67:398-404. [PubMed: 16728260] 


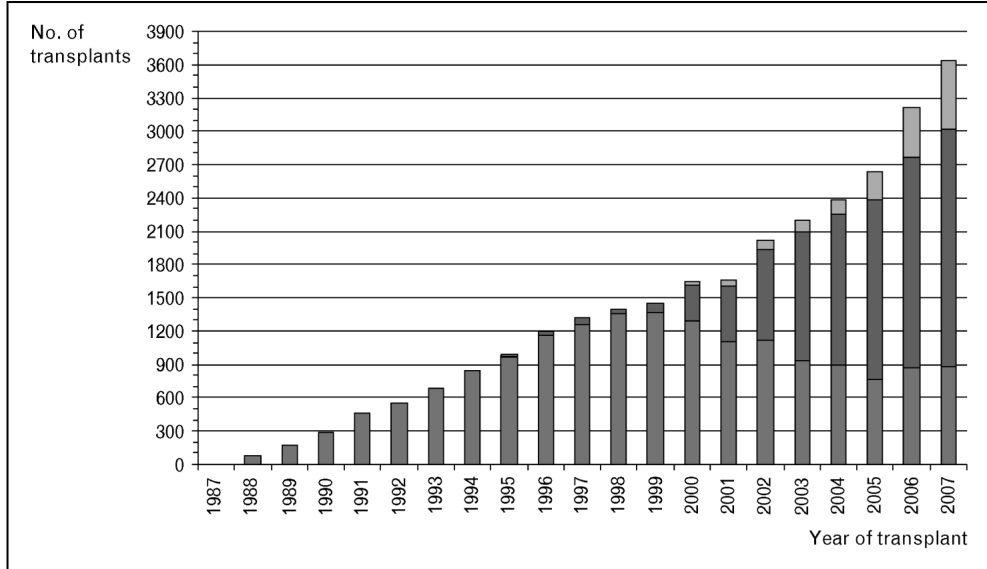

Figure 1. National Marrow Donor Program-facilitated transplants by fiscal year 1987-2007 Since the year 2000, UCBT has been rapidly increasing. $\square$ Bone marrow, $\square$ PBSC, $\square$ ) UCB. PBSC, peripheral blood stem cell; UCB, umbilical cord blood; UCBT, umbilical cord blood transplantation. Reproduced with permission from Mary Halet, NMDP. 


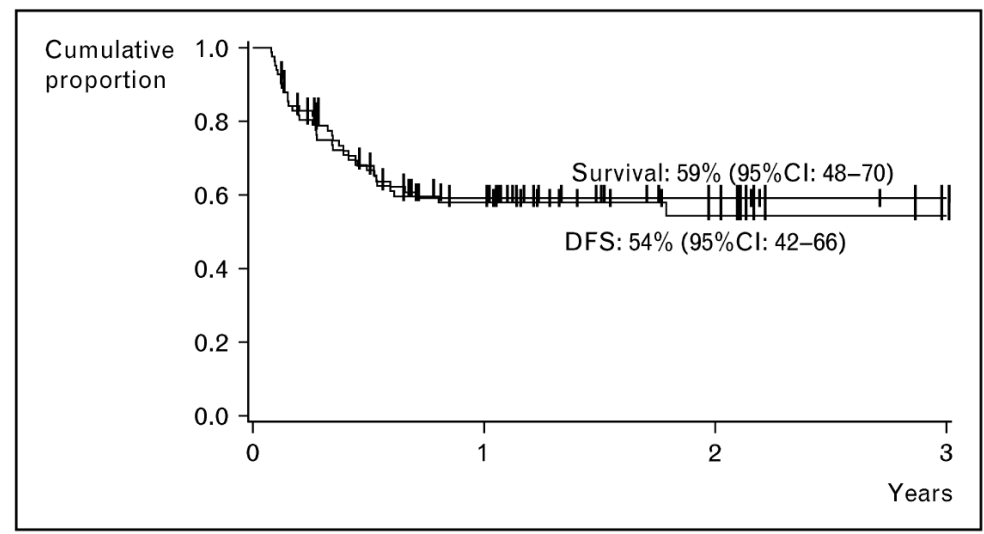

Figure 2. Survival after myeloablative double-unit umbilical cord blood transplantation $(\boldsymbol{n}=\mathbf{8 3})$ Patients were conditioned with cyclophosphamide $120 \mathrm{mg} / \mathrm{kg}$, TBI $1320 \mathrm{cGy}$, fludarabine $75 \mathrm{mg} / \mathrm{m}^{2}$ with CSA/MMF. CI, confidence interval; CSF, cyclosporine-A; DFS, disease-free survival; MMF, mycophenolate mofetil; TBI, total-body irradiation. University of Minnesota data (reproduced with permission from Professor John Wagner, University of Minnesota, 2007). 


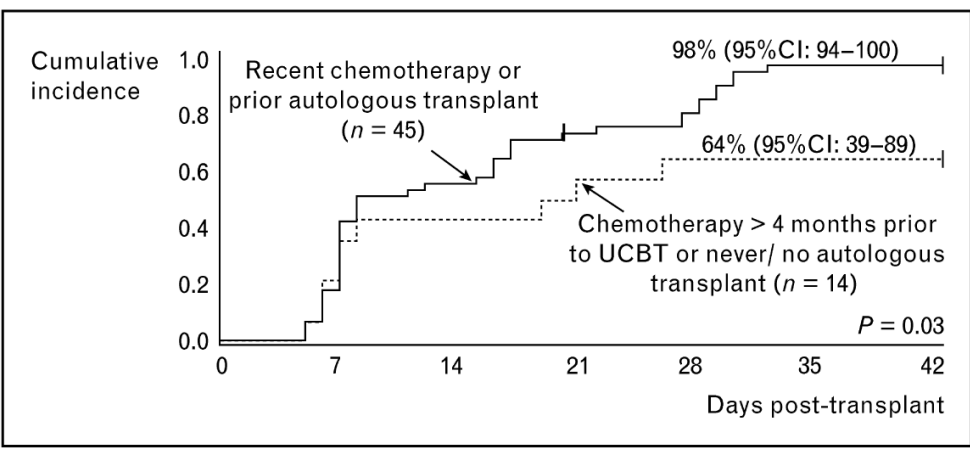

Figure 3. Association between prior chemotherapy exposure and sustained donor engraftment after nonmyeloablative umbilical cord blood transplantation

CI, confidence interval; UCBT, umbilical cord blood transplantation. University of Minnesota data. 


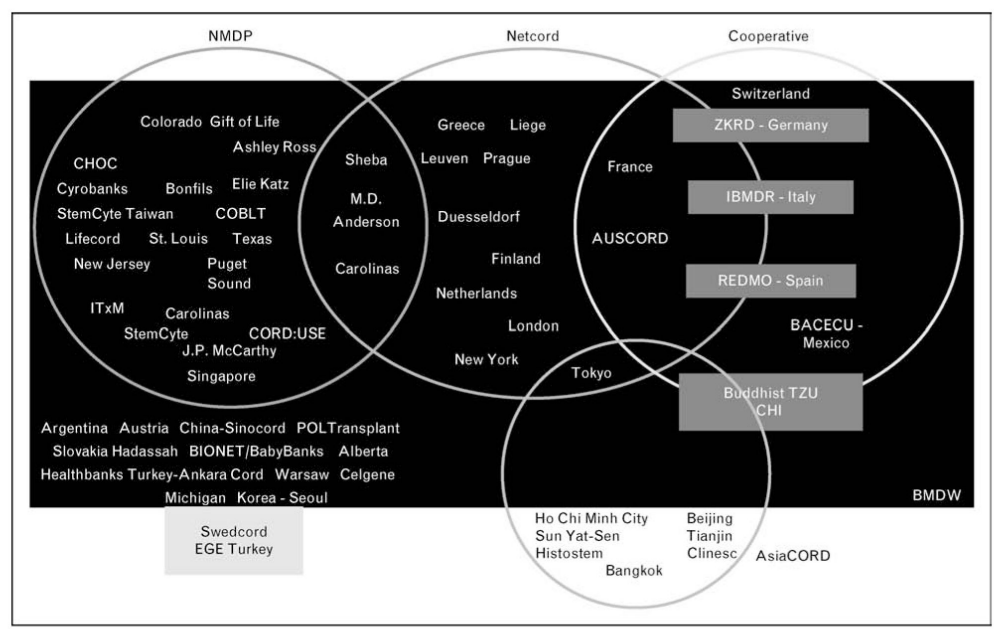

Figure 4. A schema representing the relationships between current umbilical cord blood banks BMDW, bone marrow donors worldwide; NMDP, National Marrow Donor Program Reproduced with permission from Mary Halet, NMDP, April 2008. 
Table 1

Ancestry of 48 umbilical cord blood transplantation recipients at Memorial Sloan-Kettering Cancer Center October 2005-April 2008

\begin{tabular}{lr}
\hline Patient ancestry & UCBT recipients, $\boldsymbol{n}$ \\
\hline Northwest Europe & 4 \\
Eastern Europe & 6 \\
Southern Europe & 7 \\
European Mix & 7 \\
Asian & 9 \\
African & 6 \\
Middle Eastern & 1 \\
Hispanic/Latino & 8 \\
Total & 48 \\
\hline
\end{tabular}

Patients were offered UCBT if allogeneic transplant was indicated and no suitably HLA-matched-related or unrelated volunteer donor was available. Notably, $42 \%$ of patients were of non-North Western European ancestry with $50 \%$ of patients being non-European. In addition, the four patients of North-Western European ancestry had proven or potential 9-10/10 HLA-A, B, C, DRB1, DQ allele-matched-unrelated volunteer donors but received UCB due to transplant urgency $(n=1)$ or patient preference $(n=3)$. UCB grafts were 4-6/6 HLA-matched at A and B antigens and DRB1 alleles. HLA, human leucocyte antigen; UCB, umbilical cord blood; UCBT, umbilical cord blood transplantation. 
Table 2

Relative advantages and limitations of unrelated umbilical cord blood as a hematopoietic stem cell source as compared with transplantation with unrelated volunteer donors

\begin{tabular}{ll}
\hline $\begin{array}{l}\text { Advantage of UCB } \\
\text { Rapid access without the problem of donor availability } \\
\text { (admit revolves around patient) }\end{array}$ & $\begin{array}{l}\text { Comparison with URD } \\
\text { Ability to reschedule easily }\end{array}$ \\
Reduced requirement for HLA match at high resolution & Advantage over URD \\
Less severe GVHD with chronic GVHD easier to treat ${ }^{a}$ & Major advantage over URD \\
Preserved graft-versus-leukemia effect & Major advantage over URD \\
Potential to build inventory from all racial groups & Similar to URD \\
Limitation of UCB & Major advantage over URD \\
$\begin{array}{l}\text { Limited cell dose } \\
\text { Limited inventory to enable at least 4/6 HLA matches } \\
\text { of adequate dose for patients of all races }\end{array}$ & Comparison with URD \\
$\begin{array}{l}\text { Potential for variable unit quality at thaw } \\
\text { Inability to obtain additional collections from donor } \\
\text { and naive immune system }\end{array}$ & Major disadvantage over URD \\
\hline
\end{tabular}

GVHD, graft-versus-host disease; HLA human leucocyte antigen; UCB, umbilical cord blood; URD, unrelated donor.

${ }^{a}$ Not yet examined for acute GVHD. 


\section{Table 3}

Comparison of outcomes after 8/8 HLA allele-matched unrelated donor bone marrow transplantation and 4-6 A, B antigen, DRB1 allele-matched umbilical cord blood transplantation in children with acute leukemia [13**]

\begin{tabular}{lcccc}
\hline HSC source & TRM $(\%)$ & Relapse $(\%)$ & DFS $(\%)$ & Overall survival $(\%)$ \\
\hline 8/8-matched bone marrow $(n=116)$ & 19 & 41 & 38 & 45 \\
UCB $(n=503)$ & & & & \\
$6 / 6$ & 6 & 34 & 60 & 63 \\
$5 / 6>3.0 \times 10^{7} \mathrm{NC} / \mathrm{kg}$ & 29 & 31 & 41 & 45 \\
$5 / 6<3.0 \times 10^{7} \mathrm{NC} / \mathrm{kg}$ & 43 & 21 & 37 & 36 \\
$4 / 6$ & 49 & 20 & 33 & 33 \\
\hline
\end{tabular}

Survival data are reported at 5 years after transplant. DFS, disease-free survival; HSC, hematopoietic stem cell; TRM, transplant-related mortality; UCB, umbilical cord blood. 